\title{
Diagnóstico de la política y prácticas que condicionan el acceso y permanencia de los planteles de Bachilleres de Chiapas al Sistema Nacional de Bachillerato
}

\section{Diagnosis of the policy and practices that condition the access and permanence of the Chiapas Baccalaureate campuses to the National Baccalaureate System}

\author{
ELIZONDO-ZENTENO, María del Pilar†*, CRUZ-RUEDA, Elisa y NANGULLASMÚ-SÁNCHEZ, \\ Lizbeth Alondra \\ Universidad Autónoma de Chiapas, Facultad de Humanidades Campus VI. 29050, 2a. Nte. Ote. 756, Terán, Tuxtla \\ Gutiérrez, Chis.
}

ID $1^{\mathrm{er}}$ Autor: María del Pilar, Elizondo-Zenteno / ORCID: 0000-0003-2749-2095, Researcher ID Thomson: S-79562018, CVU CONACYT ID: 219259

ID $1^{\text {er }}$ Coautor: Elisa, Cruz-Rueda / ORCID: 0000-0002-6339-1518, arXiv Author ID: 2799911, CVU CONACYT ID: 77927

ID $2^{\text {do }}$ Coautor: Lizbeth Alondra, Nangullasmú-Sánchez / ORCID: 0000-0002-4758-8214M, Researcher ID Thomson: S8436-2018, CVU CONACYT ID: 504590

DOI: $10.35429 / J B E .2019 .7 .3 .1 .7$

Recibido 15 de Enero, 2019; Aceptado 28 de Marzo, 2019

Resumen

El presente artículo analiza el diagnóstico de la implementación de la normatividad de la Educación Media Superior para el acceso y permanencia al Sistema Nacional de Bachillerato (SNB) en los planteles del Colegio de Bachilleres de Chiapas (COBACH). Para ello, se caracterizó el contexto en el que se implementó la Reforma Integral de la Educación Media Superior en los planteles del COBACH, asimismo, se identificaron las fortalezas y áreas de oportunidad de las políticas y gestión implementadas por la Administración Central del COBACH para impulsar el SBN. Para la investigación, se utilizó el método descriptivo, que parte del análisis sistémico del pasado para explicar los acontecimientos contemporáneos; mientras que la política pública educativa y la estructura del SNB se estudió con el método formal-legal. También Se utilizaron métodos y técnicas empíricas para el análisis documental, asimismo, se aplicaron técnicas e instrumentos para la recolección de datos de carácter cuantitativo y cualitativo. La investigación buscó brindar elementos académicos a las autoridades y personal del $\mathrm{COBACH}$, que permitan la toma de decisiones en sus procesos de acreditación, así también, la formación de recursos humanos en el proceso de su formación.

Políticas públicas educativas, Sistema Nacional de Bachillerato, Calidad educativa

\begin{abstract}
This article analyzes the diagnosis of the implementation of the regulations of Higher Secondary Education for access and permanence to the National Baccalaureate System (SNB) in the schools of the Baccalaureate School of Chiapas (COBACH). To this end, the context in which the Integral Reform of Higher Secondary Education was implemented in the COBACH campuses was also characterized, as well as the strengths and areas of opportunity of the policies and management implemented by the $\mathrm{COBACH}$ Central Administration to promote the SBN. For the investigation, the descriptive method was used, which starts from the systemic analysis of the past to explain contemporary events; while the educational public policy and structure of the SNB was studied with the formallegal method. Empirical methods and techniques were also used for documentary analysis, as well as techniques and instruments for quantitative and qualitative data collection. The research sought to provide academic elements to the authorities and staff of $\mathrm{COBACH}$, which allow decision making in their accreditation processes, as well as the training of human resources in the process of their training.
\end{abstract}

Public educational policies, National Baccalaureate System, Educational quality

Citación: ELIZONDO-ZENTENO, María del Pilar, CRUZ-RUEDA, Elisa y NANGULLASMÚ-SÁNCHEZ, Lizbeth Alondra. Diagnóstico de la política y prácticas que condicionan el acceso y permanencia de los planteles de Bachilleres de Chiapas al Sistema Nacional de Bachillerato. Revista de Educación Básica. 2019. 3-7: 1-7.

*Correspondencia al Autor (Correo Electrónico: mpzenteno1 @ hotmail.com)

$\uparrow$ Investigador contribuyendo como primer autor. 


\section{Introducción}

En los últimos tiempos hablar de la evaluación y acreditación de la práctica educativa tiene sus antecedentes en las políticas implementadas a nivel internacional y nacional, estas políticas establecidas como estrategias que buscan impulsar los procesos de evaluación y acreditación en los contextos escolares de los diferentes niveles educativos, en este caso hablamos de los procesos impulsados en el nivel medio superior denominado Sistema Nacional del Bachillerato (SNB) en México.

Para cumplir con los indicadores que SNB evalúa se requiere realizar todo un proceso de gestión, que implica una transformación institucional y educativa así como la implementación de una cultura de la evaluación. La investigación permitió identificar la estructura organizacional del Colegio de Bachilleres (COBACH) para el impulso del Padrón de Buena Calidad del Sistema Nacional de Educación Media Superior PBC-SiNEMS, y mostrar las fortalezas y áreas de oportunidad y con ello tener la oportunidad de dar sugerencias para las mejoras del proceso.

\section{La Gestión y la transformación Institucional}

En América Latina, se ha pasado de la perspectiva de la administración a la de gestión, autores como Pozner, Barrios y Lemus, definen a ambas como antagónicas, a la administración como una práctica de control y racionalización sistémica y sistemática y a la gestión como la forma en que cada organización o grupo de sujetos deciden llegar a las metas que se plantean, pero bajo un proceso controlado, racionalizado y sistemático. Es importante señalar que no habría tal antagonismo si se reconociera que existen distintos tipos de administración y de gestión. La gestión no es un concepto más genérico que administración, su práctica va mucho más allá de la mera ejecución de instrucciones que vienen del centro. El concepto gestión connota tanto las acciones de planificar, las de administrar, como las que se realizan por ajustes del contexto, tanto interno (lo constituyen las personas que son los miembros de la organización) como externo (por el entorno que la rodea). De ahí que pueda entenderse a la organización como un sistema que opera dentro de un contexto externo con el cual interactúa permanentemente (Casassus, 2000).
Ahora bien, si se relaciona todo lo anterior con la educación se puede observar que debido a las transformaciones estructurales que los sistemas educativos están permanentemente sometidos, se puede retomar el concepto de gestión, proveniente del mundo empresarial, como una forma de dar respuesta a dichas transformaciones, ya sea a nivel macro o micro. A nivel macro podemos hablar de gestión educativa o educacional, como aquella disciplina encargada de la gestión global de los sistemas educacionales y de la creación de políticas educativas tomando como referente el marco curricular nacional, mientras que a nivel micro podemos situar a la gestión escolar o institucional como aquella disciplina que enfatiza principalmente generar políticas concretas considerando el contexto interno de la organización, así como las características de la comunidad escolar de la institución educativa (Vera, 2006).

La gestión escolar o institucional, asimismo, se refiere a la incertidumbre originada por los cambios de los contextos de intervención, de la imposibilidad de continuar considerando a los docentes y los funcionarios como meros ejecutores, cuando en realidad son actores que toman decisiones permanentemente; de esta forma la gestión está relacionada con incertidumbre, tensiones, ambigüedades y conflictos inevitables. Por tanto, no se trata solo de efectuar un plan o de planificar sobre el papel un grupo de actividades, la planificación articula los procesos técnicos y prácticos para recuperar el sentido y la razón de ser de la gobernabilidad, del mejoramiento continuo de la calidad, equidad y la pertinencia de la educación de todas sus dependencias administrativas, docentes, programas educativos, procesos de enseñanza aprendizaje e infraestructura (Elizondo, 2008).

Es claro que la gestión de las Instituciones de Educación, que se ha venido dando con base en estos planteamientos, se pudiera considerar como tradicional, lo que hace necesario que hoy en día el establecimiento de un nuevo paradigma, un cambio que permita a las instituciones adaptarse a su contexto actual como plantea Trist B. (2004): el paradigma social. 
Con respecto al cambio de paradigma que lleva a justificar un nuevo modelo de gestión en las Instituciones de Educación Superior (IES), Pilar Pozner (1995) señala que para poder adaptarlo en la institución es indispensable poner en práctica la gestión escolar o institucional; tengan un marco de gestión que les permita un adecuado equilibrio de márgenes de autonomía. La participación de la comunidad, el apoyo institucional y la regulación normativa. Por ello es indispensable articular las estructuras y procesos mediante los cuales se realizan las funciones de gestión dentro y fuera de las instituciones, lo que involucra a cada escuela y a la institución en su conjunto.

Pozner enumera las dimensiones que trabajan la gestión escolar o institucional (2000: 23-24):

1. Dimensión pedagógico-curricular: constituyen las finalidades y objetivos que explicitan la razón de ser de la institución educativa en la sociedad.

2. Dimensión comunitaria: explicita las relaciones establecidas entre la sociedad y la escuela, haciendo especial énfasis en la vinculación entre la comunidad local y la escuela.

3. Dimensión administrativo-financiera: considera la obtención, distribución, articulación y optimización de los recursos.

4. Dimensión organizacional-operativa: busca generar la articulación de las dimensiones pedagógico-curricular, comunitaria y administrativo-financiera.

El análisis de la implementación del PBC-SiNEMS como estrategia para la transformación institucional del COBACH se apoyó en las cuatro dimensiones de gestión escolar propuestas por Pilar Pozner, quien desarrolla su trabajo en el contexto latinoamericano. Las dimensiones de la gestión permitieron analizar la gestión implementada en el COBACH para lograr que sus planteles accedan al Padrón.
En el proceso de análisis se consideraron las diferencias contextuales de las escuelas mexicanas, concretamente las chiapanecas, en donde existen diferencias abismales entre los planteles ubicados en el ámbito urbano y los que se encuentran en el rural. Por ese motivo se considera necesario trabajar, junto con las dimensiones de gestión planteadas por Pozner, con una quinta dimensión de análisis, denominada caracterización básica, desarrollada por Elizondo (2008), quien invita a presentar datos de los principales actores entre los que se encuentran el subsistema, el tipo de contratación y las acciones que desarrollan para impulsar del acceso y permanencia de sus planteles en el PBC-SiNEMS, entre otros.

La investigación buscó analizar el modelo de gestión utilizado en la institucionalización del PBC-SiNEMS, así como el nivel de avance de los planteles del COBACH en el Padrón. Sin duda identificar la gestión que realizan los directivos, así como las condiciones contextuales que benefician $u$ obstaculizan el proceso, permitirá ubicar las áreas de oportunidad del COBACH y contar con información confiable para diseñar un proceso de intervención que contribuya a elevar el número de planteles acreditados.

\section{Metodología de Investigación}

La investigación tiene un enfoque estructuralista, lo cual hizo posible analizar las dimensiones de la gestión escolar, tanto a nivel de estructura institucional, como en la percepción de los actores que la implementan en los planteles: docentes y directivos.

La investigación se centró en las instituciones de educación media superior (EMS) con financiamiento público que por el número de planteles y matrícula fueran representativos de la EMS ofertada en el estado de Chiapas. Uno de los criterios de inclusión establecido consistió en que los planteles fueran evaluables ante el Padrón. Por eso, para asegurar el estatus de dichos centros, se verificó que contarán con planes y programas de estudio dictaminados como pertinentes por el Consejo para la Evaluación de la Educación del Tipo Medio Superior (COPEEMS), documento que avala la adopción de la Reforma Integral de la Educación Media Superior (RIEMS) y el SNB. 
Fue importante que los centros escolares participantes fueran evaluables como planteles convencionales en la regla general para el acceso al PBC-SiNEMS plasmado en el Manual para evaluar planteles que solicitan ingresar o permanecer en el Padrón de Buena Calidad del Sistema Nacional de Educación Media Superior, Versión 3.0 y 4.0 (COPEEMS, 2017), por lo cual se verificó que su matrícula fuera igual o superior a los 300 estudiantes. Es importante aclarar que los planteles con menos de 300 alumnos son evaluados con parámetros alternos, sobre los cuales se brindó información general en el apartado 2.2.2. Y aunque los planteles alternos no formaron parte del interés de estudio se presentó información para caracterizar el proceso de acreditación del PBCSiNEMS.

En resumen, los planteles seleccionados para participar en la investigación cumplieron con los siguientes criterios de inclusión:

- Financiamiento público.

- Planes de estudio con dictamen de pertinencia emitido por el Consejo para la Evaluación de la Educación del Tipo Medio Superior (COPEEMS).

- Matrícula superior a 300 estudiantes.

- Evaluables con el Manual para evaluar planteles convencionales que solicitan el ingreso y la promoción al PBC-SiNEMS (Versión 3.0 y 4.0).

\section{Universo de estudio}

De acuerdo al Cuestionario Electrónico 911 SEP, en Chiapas existían 977 planteles de EMS en el ciclo escolar 2014-2015. Además la información del Cuestionario 911 permitió identificar al COBACH como el subsistema con mayor número de matrícula y planteles en el estado, situación por la cual se decidió realizar la investigación en el Colegio. Se identificó a 93 planteles que cumplieron con los criterios de inclusión los planteles se encuentran distribuidos por toda la geografía chiapaneca.
Por la dispersión geográfica y los recursos destinados a la investigación se concibió poco factible realizar la investigación en las nueve coordinaciones del $\mathrm{COBACH}$, situación que repercutió en la propuesta de una investigación en fases por el elevado número de planteles que cumplieron con los criterios establecidos. Sin embargo, cuando se valoraron el tiempo y recursos destinados al estudio, fue imposible considerar a todos, por lo que se espera que el trabajo aquí presentado no sea el único $\mathrm{y}$, posteriormente, con la misma metodología, se puedan desarrollar procesos de investigación que permitan cubrir un mayor número de planteles.

\section{Diseño de los instrumentos de recolección de información}

La investigación consideró para la recolección de datos tres estrategias (FODA):

1) Consulta documental de fuentes secundarias.

2) Cuestionario.

3) Análisis de Fortalezas, Oportunidades, Debilidades y Amenazas.

La primera de ellas permitió generar información referente al neoinstitucionalismo, así como caracterizar la gestión educativa y escolar. Por su parte, el cuestionario y el análisis FODA apoyaron la recolección de información para el análisis de las dimensiones de la gestión escolar.

\section{Diseño del cuestionario}

El cuestionario denominado: Opinión docente de las dimensiones de gestión que impulsa PBC-SiNEMS, fue diseñado con el propósito de generar un acercamiento a las opiniones de docentes del COBACH respecto al contexto en el que se implementa la gestión escolar que impulsan el acceso y permanencia de los planteles del COBACH al PBC-SiNEMS. Para su construcción se consideró el Manual de Gestión para Directores de Instituciones Educativas de la Organización de las Naciones Unidas para la Educación, la Ciencia y la Cultura (UNESCO) y los criterios establecidos por el Consejo para la Evaluación de la Educación del tipo Medio Superior para el acceso y permanencia de los planteles al PBCSiNEMS, establecidos en el Manual 3.0, así como en su actualización en el 4.0.

ELIZONDO-ZENTENO, María del Pilar, CRUZ-RUEDA, Elisa y NANGULLASMÚ-SÁNCHEZ, Lizbeth Alondra. Diagnóstico de la política y prácticas que condicionan el acceso y permanencia de los planteles de Bachilleres de Chiapas al Sistema Nacional de Bachillerato. Revista de Educación Básica. 2019 
En el proceso de diseño se realizó la operacionalización de las variables, entendida como "el paso de una variable teórica a indicadores empíricos verificables y medibles en ítems o equivalentes" (Sampieri, Fernández y Baptista, 2014: 221).

El cuestionario previo al proceso de validación estaba conformado por 40 ítems, agrupados en cuatro dimensiones de la gestión escolar, las cuales se describen a continuación (Pozner, 1997 y Casassus, 1999):

1. Dimensión organizacional-operativa: se promueve la implementación de normas explícitas e implícitas, establece una estructura institucional y se designan responsabilidades a los diferentes actores, además se valoran las capacidades personales y grupales que permiten un desarrollo institucional autónomo.

2. Dimensión administrativo-financiera: se cuenta con buen manejo de recursos económicos, humanos y se implementan procesos técnicos, de tiempo, seguridad, higiene y control de información; a su vez se da seguimiento a la aplicación de normas y supervisión de funciones de forma adecuada.

3. Dimensión pedagógico-curricular: existen estrategias didácticas, las cuales se reflejan en buenas prácticas pedagógicas, mediante la planeación, evaluación del proceso de enseñanza y aprendizaje; se brindan servicios de apoyo a los estudiantes, además se promueve la actualización continua del personal docente y directivo.

4. Dimensión comunitaria: existe relación de la escuela con el entorno y se promueve la relación con padres de familia, autoridades, organizaciones y redes de apoyo; además se busca dar respuesta a las necesidades de la comunidad.

Después del pilotaje, de 40 ítems que fueron considerados en su aplicación inicial se eliminaron dos y fue suprimida la dimensión comunitaria, resultado del proceso de validación de la fiabilidad del instrumento a través del coeficiente de fiabilidad Alfa de Cronbach.
La aplicación de este estadístico permitió valorar el instrumento en su conjunto, sus 38 ítems, logrando un puntaje de 0.912 , que indica que el instrumento tiene buena consistencia interna (Oviedo y Campo-Arias, 2005).

\section{Resultados de la Investigación}

\section{Dimensión organizacional-operativa}

La acreditación del Sistema Nacional de Bachillerato exige evidencias de que los procesos se han implementado en cada una de las dimensiones, en el caso de la dimensión organizativa-operativa es necesario fortalecer los procesos para favorecer el acceso de los planteles al SNB por lo que el $88.4 \%$ de los docentes y directivos plantean, que es indispensable la implementación de la Normatividad en la dependencia en cuanto a:

$\Rightarrow$ Idoneidad del personal docentes y directivo.

$\Rightarrow$ Trabajo en academias.

$\Rightarrow$ Plan de mejora continua.

$\Rightarrow$ Asignación de materiales.

$\Rightarrow$ Servicios escolares en relación al registro de competencias de MCC.

\section{Dimensión administrativa-financiera}

El proceso de acreditación considera dos aspectos relacionados a esta dimensión, la de los procesos y la asignación de recursos financieros.

1. En relación a los procesos:

- La aplicación de las tecnologías de la información y la comunicación (TIC) y tecnologías del aprendizaje y el conocimiento (TAC) entre los actores educativos.

- La aplicación del control de asistencias del personal.

- Cumplimiento puntal del calendario escolar.

- Ejecución de los horarios de clases. 
- Prestación de servicios bibliotecarios.

2. Asignación de recursos financieros:

- Otorgamiento de becas.

- Mantenimiento y el acceso a la infraestructura tecnológica como: aulas, laboratorios, tecnologías, sanitarios, rampas, espacios de tutorías y orientación, áreas deportivas, culturales y recreativas.

Es importante recalcar que tres cuartas partes de la población participante consideran que la gestión no se lleva de manera adecuada.

\section{Dimensión Pedagógica-curricular}

En relación a esta dimensión el 52\% de los docentes comentaron en relación a los indicadores evaluables que es indispensable reforzar:

$\Rightarrow$ Lo referente al conocimiento de los planes y programas de estudio en la comunidad escolar.

$\Rightarrow \mathrm{El}$ manejo de estrategias implementadas en clase para el desarrollo de competencias genéricas y disciplinares.

$\Rightarrow$ La diversificación de las estrategias de evaluación del aprendizaje; autoevaluación, coevaluación y heteroevaluación.

$\Rightarrow$ La prestación de servicios de tutorías y orientación y la participación de especialistas en casos especiales que así lo ameriten.

$\Rightarrow$ La inserción de los egresados a estudios de educación superior y al ámbito laboral.

$\Rightarrow$ El trabajo de las academias en los procesos de reestructuración curricular, diseño de materiales didácticos e instrumentos de evaluación.

\section{Dimensión de la gestión escolar}

Esta dimensión se valora a partir de los resultados de las tres dimensiones; organizacional-operativa, administrativafinanciera y Pedagógica-curricular y es la opinión de los actores referente al proceso de gestión desarrollado por los directivos del $\mathrm{COBACH}$ en los planteles educativos que participan en la investigación. El $72 \%$ de la población encuestada señala que es indispensable reforzar los procesos de gestión de las tres dimensiones.

\section{Conclusiones}

La investigación han permitido identificar dentro de las dimensiones evaluables aquellas que desde la opinión de los docentes cumplen de manera satisfactoria y las que requieren atención inmediata, revela que es indispensable el trabajo colaborativo y el visualizar toda una transformación institucional donde estos criterios evaluables formen parte ya de su quehacer diario.

La implementación de un nuevo modelo de gestión estratégica, implica toda una modificación institucional que les permita ir planteando de manera clara el camino a seguir por cada uno de los planteles que integran el $\mathrm{COBACH}$.

\section{Agradecimiento}

Todo nuestro agradecimiento al Consejo Nacional de Ciencia y Tecnología Fondo sectorial INEE-CONACYT ya que sin su apoyo esta investigación no habría sido posible.

\section{Referencias}

Casassus, J. (2000). Problemas de la gestión Educativa en América Latina (la tensión entre los paradigmas A y el tipo B). Pensamiento Educativo, 27, pp. 281-324.

Elizondo, M. (2008). Propuestas para el mejoramiento de la gestión del curso preuniversitario en la Universidad Autónoma de Chiapas. La Habana: Ministerio de Educación Superior. 
Elizondo, M., Castillejos, D. y Ovando, M. (2010). Estudios del Impacto de las políticas de evaluación y acreditación de la educación superior en México, una aproximación histórica. Presentado en el Séptimo Congreso Internacional de Educación Superior, Cuba.

Elizondo, M., Montesinos, L., Castillejos, D., Mena, A., y Barrientos, N. (2013). Transformación institucional en la educación superior en Chiapas: en busca de la calidad educativa. Tuxtla Gutiérrez: Universidad Autónoma de Chiapas.

Pozner, P. (1995). El directivo como gestor de aprendizaje escolar. Buenos Aires: Aique.

Pozner, P. (2000). Diez módulos destinados a los responsables de los procesos de transformación educativa. Buenos Aires: Ministerio de Educación /IIPE.

Trist., B. (2004). Introducción a la Administración Académica. México D.F: Universidad Autónoma Metropolitana-Unidad Xochimilco. 